\title{
MARCO TEÓRICO DE ANÁLISIS DE LOS COMPONENTES DE LOS SISTEMAS ALIMENTARIOS LOCALES (LFS)
}

Inmaculada Marques-Perez ${ }^{a^{*}}$, Estefanía Osorio ${ }^{a}$ Luzvenia Miranda ${ }^{a}$.

${ }^{a}$ Universitat Politècnica de València

\section{Resumen}

Los Sistemas Locales de Alimentación (Local Food Systems: LFS) son resultado, entre otros factores, del crecimiento de la población urbana, de los cambios recientes en las interrelaciones agricultor-consumidor, por el cambio en las demandas del consumidor; de la creciente escasez de recursos y la necesidad de las administraciones de garantizar una alimentación saludable; y también por la necesidad de los agricultores de participar mejor de la cadena de valor agroalimentaria. Representan un sistema innovador alternativo con el potencial de ofrecer un modelo de desarrollo económico socialmente más inclusivo y medioambientalmente más responsable. En las últimas décadas muchos académicos han investigado en torno a los LFS, con aportaciones en entorno a su definición, los ámbitos que deben abarcar y los agentes que deben implicar, analizando diferentes iniciativas locales. Sin embargo, aún no se ha abordado un análisis completo sobre lo que implica la definición y diseño de LFS, y la propuesta de un marco teórico de diseño y desarrollo, pero también de análisis de los LFS. Mediante una revisión sistemática de la literatura, en nuestro trabajo se pretende mejorar nuestra comprensión de los componentes clave de los LFS. El objeto es identificar componentes, interrelaciones, e indicadores de análisis y seguimiento que permitan establecer un marco teórico no sólo para la definición sino también para el análisis, revisión y mejora de los LFS.

\section{Palabras clave}

Sistemas alimentarios locales, sostenibilidad, estrategias urbanas alimentarias

\section{Introducción}

En las últimas décadas, según Smaal, et al. (2020) los académicos han estado pidiendo cada vez más, soluciones holísticas y políticas alimentarias basadas en lo local, que promuevan soluciones contra la escasez de alimentos, la necesidad de dietas saludables y sostenibles y contra el desperdicio alimentario. Para Jones \& Hills (2021) esta atención emergente refleja la conciencia del papel distintivo que las ciudades pueden jugar para facilitar una transformación sistémica del sistema alimentario. Además como destaca Galimberti et al. (2019).la pandemia del COIVD-19 ha puesto de relieve entre otros aspectos, el papel estratégico específico de las ciudades en el desarrollo de sistemas alimentarios sostenibles y la promoción de hábitos alimentarios saludables

Moragues y Moran (2015) destacan el papel de las ciudades como espacios donde ir moldeando estos nuevos sistemas de gobernanza alimentaria, creando espacios de debate conjunto entre la sociedad civil, los actores privados, y la administración pública local para la construcción de estos nuevos sistemas alimentarios. Según Candel (2020), desde la creación en 1991, del Consejo Alimentario Municipal de Toronto, las administraciones locales han ido registrando distintas iniciativas locales que en el 2015 confluyeron en el Pacto de Política Alimentaria Urbana de Milán de 2015 (MUFPP. El Pacto está firmado actualmente por más de 200 ciudades. La firma del pacto constituye un compromiso internacional y es a la vez una guía de actuación para los municipios firmantes en seis áreas temáticas: gobernanza; nutrición y alimentación sostenible; equidad social y económica; producción alimentaria; provisión y distribución alimentaria desperdicio alimentario.

Sin embargo, se observa en distintos estudios empíricos como los de Sonnino (2019), Doernberg, Horn, Zasada, \& Piorr (2019) y Candel (2020) las actividades de política alimentaria urbana están todavía muy fragmentadas y, a menudo, se basan en iniciativas individuales dentro de la administración, sin una política bien definida por los gobernantes. En este sentido, la definición de políticas alimentarias urbanas integradas y su implementación a través de Estrategias Alimentarias Urbanas siguen siendo una excepción en muchas ciudades, incluso aquellas firmantes del Pacto de Milan, y cuando existen, es difícil evaluar su éxito. De una parte, porque todavía es pronto para poder ver el resultado de las políticas, de otra parte, porque hay una cierta dificultad para identificar los sectores y componentes, que deben contemplar, los actores que intervienen, y también carencia en la definición de indicadores que permitan realizar tal seguimiento. Es por eso por lo que como destaca Schreiber, et al. (2021) en la actualidad, la falta de un marco metodológico y una agenda de investigación coherentes limita el potencial para comparar lo realizado en diferentes ciudades y regiones, así como para acumular conocimientos.

El presente trabajo es una revisión sistemática de bibliografía encaminada precisamente a la definición de elementos que integran o deben integrar los sistemas agroalimentarios, así como la definición de indicadores que permitan el adecuado seguimiento del funcionamiento de estos. La revisión parte de una búsqueda en Web of Science (WOS). 


\section{Objetivos}

Mediante una revisión sistemática de la literatura, en nuestro trabajo se pretende mejorar la comprensión de los componentes clave de los LFS. El objeto es identificar componentes, interrelaciones, y conexiones, e indicadores de análisis y seguimiento que permitan establecer un marco teórico no sólo para la definición sino también para el análisis, revisión y mejora de los LFS.

\section{Metodología}

Se ha llevado a cabo una revisión de la bibliografía académica de los últimos 20 años. La búsqueda se ha realizado en WOS, y por tanto publicaciones o manuscritos sujetos a revisión de pares, si bien sin establecer restricciones en cuanto al tipo de manuscrito, incluyendo revistas, libros, tampoco respecto de la fecha de publicación a la hora de realizar la búsqueda. La sistemática seguida ha sido:

- Establecer los descriptores para realizar las búsquedas: "local food system", "sustainable", "urban food policy" anf "urban food strategies" todos como tema, y realizando refinos: 1) local food system, sustainable, urban food startegies; 2) urban food system, sustainable, urban food policy. Se hizo una búsqueda considerando el título: urban food system (172), sustainable (54)

- Criterios de inclusión: artículos que realizaban aportaciones sobre los sistemas alimentarios urbanos desde una perspectiva global de conjunto del sistema

- Se propuso la revisión de los últimos 20 años, aunque en la búsqueda no se estableció ninguna restricción.

\section{Resultados}

De la búsqueda resultaron los siguientes ítems:

- Búsquedas: "local food system", "sustainable", "urban food policy" anf "urban food strategies" como tema, y realizando refinos: 1) local food system (10.564 resultados), sustainable (2.055), urban food startegies (82); 2) urban food system (4.143), sustainable (040), urban food policy (326). Se hizo una búsqueda considerando el título: urban food system (172), sustainable (54)

- A partir del abstract de los artículos resultantes de las diferentes búsquedas y los correspondientes refinos, se seleccionaron aquellos artículos que realizaban aportaciones sobre los sistemas alimentarios urbanos desde una perspectiva global de conjunto del sistema

- Se propuso la revisión de los últimos 20 años, si bien en las búsquedas no se encontraron artículos anteriores al 2000 que realizaran aportaciones sobre sistemas alimentarios urbanos. La primera referencia aparece en 2004 y es referida a Ontario (Canadá).

En la revisión se reconoce la creciente preocupación por el diseño de agendas de desarrollo urbano alternativas, a partir de las interconexiones entre los objetivos económicos, medioambientales, de equidad social, de salud, etc. Aunque no siempre desde la perspectiva de conjunto del sistema alimentario, los aspectos que se destacan en las nuevas políticas urbanas en este sentido contemplan aspectos entre otros, como: la promoción del consumo y la producción responsables, la conservación de la agrobiodiversidad(Altieri \& Toledo, 2011), la redefinición de las interrelaciones en la cadena de suministro y las nuevas redes de consumo, la preocupación creciente por la salud y la necesidad de contar con dietas saludables, la minimización del desperdicio, etc.

En relación a los actores, incluyen tanto a la sociedad civil, como a actores privados y a los gobiernos locales, para elaborar las estrategias hacia futuros alimentarios más sostenibles, experimentando con nuevas formas de gobernanza alimentaria. Entre los actores privados, aquellos involucrados en la producción, procesamiento, distribución y consumo de productos alimenticios a escala territorial (agricultores, intermediarios, procesadores). La sociedad civil incluyendo ONG's, y a nivel de gobierno local, instituciones agrícolas, autoridades locales, etc.

Loa ámbitos en los que se agrupan los distintos elementos y las acciones que promueven son 4: dimensión económica, dimensión ambiental, dimensión socio-cultural y la dimensión salud. En cada dimensión, para los diferentes actores se definen aquellos criterios que se considera son determinantes de la propia condición y naturaleza del sistema alimentario como sistema local, sostenible, integrado, que debe promover y desarrollar determinadas actuaciones a través de las nuevas formas de gobernanza.

La definición de indicadores para el seguimiento y la revisión debe realizarse en base a las posibilidades de registros y datos existentes, o cuya generación y registro sea factible en términos de calidad de la información y su aprovechamiento.

A modo de ejemplo, se incluye a continuación los detalles resumidos de la definición de la dimensión económica de los sistemas agroalimentarios locales, a partir de la revisión realizada. Las referencias de bibliografía se han simplificado y ajustado a la presente acta, si bien la revisión es sumamente exhaustiva y detallada. 


\begin{tabular}{|c|c|c|}
\hline \multicolumn{3}{|c|}{ DIMENSIÓN ECONÓMICA } \\
\hline CRITERIOS & $\begin{array}{l}\text { GOBERNANZA } \\
\text { PRIVÁBLICA/ONG's }\end{array}$ & INDICADORES \\
\hline \multicolumn{3}{|c|}{ ACTORES DE LA CADENA ALIMENTARIA } \\
\hline \multicolumn{3}{|c|}{ SECTOR PRODUCTOR } \\
\hline \multirow{3}{*}{$\begin{array}{l}\text { Apoyo a la producción } \\
\text { local (Vieira, et al. 2018) }\end{array}$} & \multirow{2}{*}{$\begin{array}{l}\text { Mercados locales de agricultores } \\
\text { (López, et al., 2018) }\end{array}$} & $\mathrm{N}^{\circ}$ de agricultores que comercializan directamente \\
\hline & & $\begin{array}{l}\text { Ayudas económicas a la producción ecológica (López, } \\
\text { D., et.al 2018) }\end{array}$ \\
\hline & $\begin{array}{l}\text { Pagos de compensación a los } \\
\text { agricultores (Galli et al., 2020) }\end{array}$ & $\begin{array}{l}\text { Ayudas en los sectores agrícola y forestal y en las } \\
\text { zonas rurales (Galli et al., 2020) }\end{array}$ \\
\hline $\begin{array}{l}\text { Producción agricola urbana } \\
\text { (Vieira et al., 2018) }\end{array}$ & $\begin{array}{l}\text { Apoyo al desarrollo de huertos } \\
\text { urbanos (Sonnino, et al., 2019) }\end{array}$ & $\begin{array}{l}\% \text { de tierras agrícolas en zona urbana, } \mathrm{N}^{\circ} \text { de } \\
\text { agricultores }\end{array}$ \\
\hline $\begin{array}{l}\text { Diversidad de sistemas de } \\
\text { producción de alimentos } \\
\text { (Vieira et al., 2018) }\end{array}$ & $\begin{array}{lll}\begin{array}{l}\text { Sistemas } \\
\text { alternativos }\end{array} & \text { de } & \text { producción } \\
\end{array}$ & $\begin{array}{l}\text { Análisis y encuestas sobre producción y } \\
\text { comercialización de alimentos regionales (Ilieva, } \\
\text { 2017). }\end{array}$ \\
\hline \multirow[t]{2}{*}{$\begin{array}{l}\text { Calidad de vida para los } \\
\text { trabajadores de la cadena } \\
\text { alimentaria }\end{array}$} & $\begin{array}{l}\text { Rentas dignas para el productor y } \\
\text { para los trabajadores }\end{array}$ & $\begin{array}{l}\text { Precios justos para productores, ingresos agrícolas } \\
\text { netos en efectivo, salario medio de trabajadores del } \\
\text { sistema alimentario (Ilieva, 2017) }\end{array}$ \\
\hline & Condiciones de vida & $\begin{array}{l}\text { Programas orientados a mejorar las condiciones de } \\
\text { vida de los trabajadores agrícolas (vivienda, atención } \\
\text { médica, servicios) (Ilieva, 2017) }\end{array}$ \\
\hline $\begin{array}{l}\text { Nuevas tecnologias } \mathrm{e} \\
\text { innovación }\end{array}$ & Proyectos de innovación & $\begin{array}{l}\mathrm{N}^{\circ} \text { de proyectos de innovación (plataformas digitales, } \\
\text { sistemas de información agraria, etc) (Ilieva, 2017) }\end{array}$ \\
\hline $\begin{array}{lr}\begin{array}{l}\text { Fomentar una } \\
\text { alimentaria }\end{array} & \text { economía } \\
\text { vibrante y diversa } & \text { regional }\end{array}$ & Apoyo a los productores locales & $\begin{array}{l}\text { Contrataciones públicas para la compra de productos } \\
\text { locales, de temporada, organicos, de comercio justo en } \\
\text { escuelas, hospitales y residencias (Sonnino, 2019) }\end{array}$ \\
\hline \multicolumn{3}{|c|}{ PROCESADO Y MANIPULACIÓN } \\
\hline \multicolumn{3}{|l|}{$\begin{array}{l}\text { Aprovisionamiento } \\
\text { prioritariamente de las } \\
\text { unidades de transformación } \\
\text { con materias primas } \\
\text { agrícolas de la región donde } \\
\text { estas son implantadas }\end{array}$} \\
\hline $\begin{array}{l}\text { Los sistemas de etiquetado } \\
\text { para la nutrición (Galli et } \\
\text { al., 2020) }\end{array}$ & $\begin{array}{lll}\text { Etiquetado } & \text { de } & \text { productos } \\
\text { ecológicos } & & \end{array}$ & 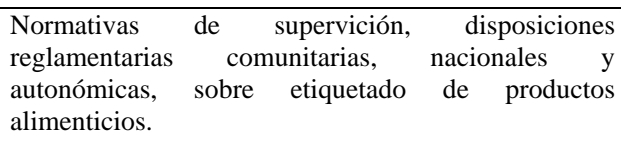 \\
\hline $\begin{array}{l}\text { Tecnologias en procesado y } \\
\text { manipulación }\end{array}$ & $\begin{array}{l}\text { Iniciativas que faciliten el acceso } \\
\text { a tecnología de procesado y } \\
\text { manipulación de alimentos }\end{array}$ & $\begin{array}{l}\text { Incorporación de tecnologías digitales en colaboración } \\
\text { con centros de investigación, universidades y empresas } \\
\text { privadas (López, et.al 2018) }\end{array}$ \\
\hline \multicolumn{3}{|c|}{ COMERCIO Y DISTRIBUCIÓN } \\
\hline \multirow[t]{2}{*}{$\begin{array}{lr}\text { Diversificar } & \text { canales } \\
\text { comerciales } & \text { (López, et.al } \\
\text { 2018) } & \end{array}$} & 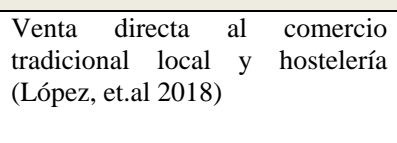 & $\begin{array}{l}\text { Total de alimentos frescos comprados } \mathrm{Kg} / \mathrm{mes}, \mathrm{N}^{\circ} \text { de } \\
\text { agricultores que comercializan directamente (Ilieva, } \\
2017 \text { ) } \mathrm{N}^{\circ} \text { de tiendas y comerciantes de alimentos } \\
\text { locales }\end{array}$ \\
\hline & $\begin{array}{l}\text { Cadena de suministro regional } \\
\text { más corta y sólida }\end{array}$ & $\begin{array}{l}\text { Nuevos modelos de negocio cooperativo, mercados } \\
\text { alimentarios y minoristas (López, D., et.al 2018) }\end{array}$ \\
\hline \multirow{2}{*}{$\begin{array}{l}\text { Acceso a infraestructuras } \\
\text { logísticas y de distribución } \\
\text { para las redes locales } \\
\text { (López, et.al 2018) }\end{array}$} & $\begin{array}{l}\text { Proyectos de acceso } \mathrm{a} \\
\text { infraestructura logistica }\end{array}$ & $\begin{array}{l}\text { Porcentaje de organización de ventas en línea, } \\
\text { organización del día de distribución, comunicación } \\
\text { (Vicente-Almazán Castro et al., 2019) }\end{array}$ \\
\hline & & $\begin{array}{l}\text { Cesión de infraestructuras logísticas para la venta de } \\
\text { productos locales (López, et.al 2018) }\end{array}$ \\
\hline $\begin{array}{l}\text { Distribución de valor más } \\
\text { equitativa a lo largo de la } \\
\text { cadena de suministro }\end{array}$ & $\begin{array}{l}\text { Crear oportunidades para que los } \\
\text { productores vendan directamente } \\
\text { a los consumidores. }\end{array}$ & $\begin{array}{l}\text { Incentivos fiscales de fomento de la comercialización } \\
\text { (López, D., et.al 2018), Índice de volatilidad de } \\
\text { precios (Ilieva, 2017) }\end{array}$ \\
\hline \multicolumn{3}{|l|}{ SOCIEDAD Y CONSUMO } \\
\hline \multirow{2}{*}{$\begin{array}{l}\text { Acceso a alimentos locales } \\
\text { y sostenibles a colectivos } \\
\text { de mayor vulnerabilidad } \\
\text { (Vieira et al., } 2018\end{array}$} & $\begin{array}{l}\text { Programas solidarios de entrega } \\
\text { de alimentos }\end{array}$ & $\begin{array}{l}\mathrm{N}^{\circ} \text { de empresas solidarias., } \mathrm{N}^{\circ} \text { de banco de alimentos, } \\
\text { reparto de alimentos a domicilio }\end{array}$ \\
\hline & Comedores sociales & $\%$ de beneficiados según colectivos \\
\hline
\end{tabular}




\begin{tabular}{|c|c|c|}
\hline \multicolumn{3}{|c|}{ DIMENSIÓN ECONÓMICA } \\
\hline CRITERIOS & $\begin{array}{ll}\text { GOBERNANZA } & \text { PÚBLICA/ } \\
\text { PRIVADA/ONG's } & \end{array}$ & INDICADORES \\
\hline \multicolumn{3}{|c|}{ ACTORES DE LA CADENA ALIMENTARIA } \\
\hline \multicolumn{3}{|l|}{ RESIDUOS } \\
\hline $\begin{array}{l}\text { Prevención y reducción de } \\
\text { residuos }\end{array}$ & $\begin{array}{l}\text { Estrategia para el consumo } \\
\text { sostenible (Galli et al.,2020) }\end{array}$ & $\begin{array}{l}\text { Educación alimentaria (por ejemplo, residuos) (Galli et } \\
\text { al.,2020), Volumen de residuos sólidos urbanos } \\
\text { (Ilieva, 2017) }\end{array}$ \\
\hline Económia cicular & $\begin{array}{l}\text { Iniciativas que incorporen la } \\
\text { importancia de reducir, reutilizar } \\
\text { y reciclar los recursos } \\
\text { alimentarios (Vieira et al., 2018) }\end{array}$ & $\begin{array}{l}\text { Porcentaje de residuos enviados para el } \\
\text { reprocesamiento, usado como alimentos para animales } \\
\text { ó materia prima para el fertilizante industrial o aportes } \\
\text { para energía sostenible e incineración. (Vieira et al., } \\
2018 \text { ) }\end{array}$ \\
\hline
\end{tabular}

\section{Conclusiones}

El trabajo aporta un marco metodológico coherente con potencial para analizar lo ya realizado en diferentes ciudades y regiones, así como para realizar un adecuado seguimiento con proyección.

\section{Bibliografía}

Altieri, M. A., \& Toledo, V. M. (2011). The agroecological revolution in Latin America: Rescuing nature, ensuring food sovereignty and empowering peasants. Journal of Peasant Studies, 38(3). https://doi.org/10.1080/03066150.2011.582947

Candel, J. J. L. (2020). What's on the menu? A global assessment of MUFPP signatory cities' food strategies. Agroecology and Sustainable Food Systems, 44(7), 919-946. https://doi.org/10.1080/21683565.2019.1648357

Doernberg, A., Horn, P., Zasada, I., \& Piorr, A. (2019). Urban food policies in German city regions: An overview of key players and policy instruments. Food Policy, 89(November). https://doi.org/10.1016/j.foodpol.2019.101782

Galimberti, A., Cena, H., Campone, L., Ferri, E., Dell’Agli, M., Sangiovanni, E., ... Labra, M. (2019). Rethinking Urban and Food Policies to Improve Citizens Safety After COVID-19 Pandemic. Frontiers in Nutrition | Www.Frontiersin.Org, 7, 569542. https://doi.org/10.3389/fnut.2020.569542

Galli, F., Prosperi, P., Favilli, E., D’Amico, S., Bartolini, F., \& Brunori, G. (2020). How can policy processes remove barriers to sustainable food systems in Europe? Contributing to a policy framework for agri-food transitions. Food Policy, 96. https://doi.org/10.1016/j.foodpol.2020.101871

Ilieva, R. T. (2017). Urban food systems strategies: A promising tool for implementing the SDGs in practice. Sustainability (Switzerland), 9(10). https://doi.org/10.3390/su9101707

Jones, M., \& Hills, S. (2021). Scaling up action on urban sustainable food systems in the united kingdom: Agenda setting, networking, and influence. Sustainability (Switzerland), 13(4), 1-13. https://doi.org/10.3390/su13042156

López, D., Alonso, N., \& Herrera, P. M. (2018). Políticas alimentarias urbanas para la sostenibilidad. Análisis de experiencias en el Estado español, en un contexto internacional.

Smaal, S. A. L., Dessein, J., Wind, B. J., \& Rogge, E. (2020). Social justice-oriented narratives in European urban food strategies: Bringing forward redistribution, recognition and representation. Agriculture and Human Values. https://doi.org/10.1007/s10460-020-10179-6

Sonnino, R, \& Spayde, J. (2014). The New Frontier? Urban strategies for food security and sustainability. In Sustainable food systems: building a new paradigm (pp. 186-205).

Sonnino, Roberta. (2019). The cultural dynamics of urban food governance. City, Culture and Society, 16(November 2017), 12-17. https://doi.org/10.1016/j.ccs.2017.11.001

Sonnino, Roberta, Tegoni, C. L. S., \& De Cunto, A. (2019). The challenge of systemic food change: Insights from cities. Cities, 85. https://doi.org/10.1016/j.cities.2018.08.008

Vicente-Almazán Castro, L., Herrera-Gil, M., \& Escobar-Cruz, M. (2019). SISTEMAS ALIMENTARIOS TERRITORIALIZADOS EN ESPAÑNA. 100 Iniciativas Locales Para Una Alimentación Responsable y Sostenible. Valencia.

Vieira, L. C., Serrao-Neumann, S., Howes, M., \& Mackey, B. (2018). Unpacking components of sustainable and resilient urban food systems. Journal of Cleaner Production, 200, 318-330. https://doi.org/10.1016/j.jclepro.2018.07.283 